\title{
Systematic interactome mapping of acute lymphoblastic leukemia cancer gene products reveals EXT-1 tumor suppressor as a Notch1 and FBWX7 common interactor
}

\author{
Sarah Daakour ${ }^{1}$, Leon Juvenal Hajingabo ${ }^{1,2}$, Despoina Kerselidou', Aurelie Devresse', Richard Kettmann',
} Nicolas Simonis ${ }^{2}$, Franck Dequiedt ${ }^{1}$ and Jean-Claude Twizere ${ }^{1 *}$

\begin{abstract}
Background: Perturbed genotypes in cancer can now be identified by whole genome sequencing of large number of diverse tumor samples, and observed gene mutations can be used for prognosis and classification of cancer subtypes. Although mutations in a few causative genes are directly linked to key signaling pathways perturbation, a global understanding of how known cancer genes drive oncogenesis in human is difficult to assess.

Methods: We collected available information about mutated genes in Acute Lymphoblastic Leukemia (ALL). Validated human protein interactions (PPI) were collected from IntAct, HPRD and BioGRID interactomics databases, or obtained using yeast two-hybrid screening assay.

Results: We have mapped interconnections between 116 cancer census gene products associated with ALL. Combining protein-protein interactions data and cancer-specific gene mutations information, we observed that 63 ALL-gene products are interconnected and identified 37 human proteins interacting with at least 2 ALL-gene products. We highlighted exclusive and coexistence genetic alterations in key signaling pathways including the PI3K/AKT and the NOTCH pathways. We then used different cell lines and reporter assay systems to validate the involvement of EXT1 in the Notch pathway.

Conclusion: We propose that novel ALL-gene candidates can be identified based on their functional association with well-known cancer genes. We identified EXT1, a gene not previously linked to ALL via mutations, as a common interactor of NOTCH1 and FBXW7 regulating the NOTCH pathway in an FBXW7-dependend manner.
\end{abstract}

Keywords: Acute lymphoblastic leukemia, Cancer genes, Interactome, Notch pathway, EXT1

\section{Background}

The identification of genes responsible for oncogenesis is a major goal in cancer research. These genes are mostly defined as "altered genes directly promoting malignant progression". After three decades of molecular cancer research, different strategies have been used to define the cancer genetic landscape. The catalogue of somatic mutations in cancer (COSMIC) (http://cancer.sanger.ac.uk) is a comprehensive resource of somatic mutations in human

\footnotetext{
* Correspondence: jean-claude.twizere@ulg.ac.be

'Laboratory of Protein Signaling and Interactions, Molecular Biology in Diseases Unit, GIGA-Research, University of Liège, Liège B-4000, Belgium

Full list of author information is available at the end of the article
}

cancer samples curated from published studies and cancer genomes sequencing [1]. As of May 31, 2015, COSMIC reports a set of 572 genes, called the cancer gene census, for which mutations are associated with cancer development. In cancer samples, it is challenging to analyze and prioritize sequential mutation accumulation events, which occur in oncogenes and tumor suppressors genes. The mutations that provide a selective growth advantage in any step of tumorigenesis (initiation, clonal expansion, tumor formation) are known as driver mutations. Out of 572 cancer gene census, about 140 genes including 71 tumor suppressors and 54 oncogenes are well-accepted as cancer driver genes because mutations in those genes 
promote tumorigenesis [2]. These numbers are not static and should increase as more cancer genomes are sequenced. Other approaches are being used to identify novel candidate cancer genes including Genome Wide Association Studies (GWAS) for the identification of cancerassociated loci [3], in vivo transposon mutagenesis screens in mice "sleeping beauty technology", for genes potentially implicated in tumorigenesis [4-6], and protein-protein interactions screens of gene products targeted by oncogenic viruses [7-9]. Integrating information from all the above resources in a "guilt-by-association" model that also considers interacting partners of cancer-associated gene products allowed prioritization of $\sim 3000$ genes potentially associated with cancer [10]. However, analyzing variations of mutations in time and space in different cancer types and subtypes (e.g., what driver genes are important for what cancer type at what stage) has been challenging. Few studies led to the discovery of a number of genes implicated in specific tumor types. As an example, children medulloblastoma tumor samples exhibit an average of 11 gene alterations compared to 55-121 in adult tumors [11], whilst lung and colorectal cancers require only 3 driver gene mutations [12]. In liquid tumors such as leukemia and lymphomas, it believed that, one of the most prevalent category of mutations involving cancer driver genes are chromosomal rearrangements such as BCR-ABL1 in chronic mylogenous leukemia (CML) [13], fusions involving nucleopins 98 and 124 and MLL gene fusions in acute myelogenous leukemia (AML) $[2,14,15]$, and TEL-AML1 and TCF3-PBX1 in acute lymphoblastic leukemia (ALL) [16-18]. These gene fusions alone are often insufficient and may require additional genetic perturbations for leukemogenesis $[19,20]$.

We previously showed that protein-protein interactions (PPI) data could be used for interpretation of expression profiles in cancer samples in order to identify and prioritize target genes and pathways [20]. Here, we used PPI mapping strategies to explore information on cancer genes frequently mutated in ALL. We highlighted mutated hub proteins interconnected in an ALL-cancer gene products network and identified novel interacting partners that link key ALL-cancer driver gene products $[21,22]$.

\section{Methods}

\section{Databases and literature PPI curation}

Information about genes containing mutations in their coding regions was retrieved from the COSMIC database, evaluated; organized and selected genes were submitted for experimental analysis. To establish a catalog of genes and mutations associated with Acute Lymphoblastic Leukemia (ALL), we used the version 71 of COSMIC, previously downloaded to a local server and we extracted data only related to ALL. We developed and implemented a procedure that automatically collects information and check the consistency of changes with the coding sequences and find the corresponding positions on clones from the human ORFeome (http://horfdb.dfci.harvard.edu/).

The retrieved information include details provided at either nucleotide or protein level (mutation syntax), sample id (portion of a tumor being examined for mutations), tissue from which the sample originated, histological classification of the sample and the Pubmed id of the article that published the study. For each gene tested for PPIs using the yeast two-hybrid, we identified mutations positions on the ORFeome clone by sequence alignment (BLAST) then we verified if the protein sequence has undergone modifications as described by mutation syntax.

Human PPIs were collected and verified from the different interactomics databases IntAct [23], HPRD [24] and BioGRID [25]. Only physical PPIs validated at least in two independent references or by two methods were considered as confident and maintained for the analysis.

\section{Network data analyses and visualization}

Network analyses and visualization of protein-protein interactions were carried out with Cytoscape software, which is a free software for visualizing, modeling and analyzing molecular and genetic interaction networks. Due to its features, Cytoscape and its plugins provide a powerful tool kit allowing to answer specific biological questions using large amounts of cellular network and molecular profiling information [26]. In our maps, the nodes represent proteins that are connected with edges representing pairwise interactions extracted from interaction databases and from our $\mathrm{Y} 2 \mathrm{H}$ experimental assay.

\section{Cell culture and transfection}

HEK293, HeLa and HeLa Notch1 $\triangle$ E-eGFP cells were cultured in DMEM supplemented with $10 \%$ fetal bovine serum (FBS), $2 \mathrm{mM}$ glutamine and penicillin/streptomycin. The same medium was used for U2OS Tet-on flp-in cells bearing isogenic transgenes encoding Notch1-Gal4. As for K562-control and K562 expressing Dll4 cells, they were grown and maintained in RPMI 1640 supplemented with $15 \%$ FBS and antibiotics. T-ALL cell lines were grown and maintained in RMPI containing 10-20 \% FBS and supplemented with antibiotics.

HEK293 cells were DNA-transfected with polyethylenimine (PEI) purchased from Sigma, reagent was dissolved in water at $1 \mathrm{mg} / \mathrm{ml}$ and preserved at $-80^{\circ} \mathrm{C}$. Transfection with PEI was performed on HEK cells cultured in DMEM at $80 \%$ confluence. Medium was changed before transfection and cells were collected $24 \mathrm{~h}$ post-transfection.

HeLa cells and HeLaN1 $\triangle$ E-eGFP cells were DNAtransfected with lipofectamine 2000 reagent (Invitrogen) according to manufacturer's instructions and collected $24 \mathrm{~h}$ post-transfection. 
SiRNA transfection was performed with Calcium Phosphate using ProFection Mammalian Transfection kit from Promega according to manufacturer's instructions on cells cultured in DMEM at 40-50 \% confluence. Medium was changed $24 \mathrm{~h}$ later and cells were collected $48 \mathrm{~h}$ posttransfection.

For experiments involving both DNA and siRNA transfections, siRNA-transfection was performed according to manufacturer's instructions and $24 \mathrm{~h}$ later after changing medium cells were transfected with DNA using lipofectamin 2000 reagent (Invitrogen) and cells were collected $24 \mathrm{~h}$ post DNA-transfection.

For proteasomal degradation inhibition, cells were treated with $10 \mu \mathrm{g} / \mathrm{ml}$ MG132 for $6 \mathrm{~h}$ before being collected.

siRNA sequences:

siEXT1: 5' -GGAUUCCAGCGUGCACAUUtt-3' siFBXW7: 5' - GCAUAGAUUUUAUGGUAAtt-3' siCtrl: 5' - GGCUGCUUCUAUGAUUAUGtt-3'

\section{qRT-PCR}

Total RNA was extracted using GeneJET RNA Purification Kit (Thermo scientific), DNaseI-treated on the column (Thermo Scientific) and reverse-transcribed with random primers (Thermo scientific). qPCR was performed using SYBER Green detection from Roche and run on Lightcycler 480 (Roche). mRNA quantification was performed relative to GAPDH housekeeping gene. Relative expression levels were calculated for each gene using the $\Delta \Delta \mathrm{Ct}$ method.

\section{Plasmids}

Open reading frames (ORF) encoding Notch1 partners (tested for Protein complementation assay) were obtained from human ORFeome v5.1 (center of cancer systems biology: CCSB) as entry clones. Human NICD plasmid, was obtained from Addgene. FBXW7 $\alpha$ expressing vector was kindly provided by Dr. E. Dejardin from the laboratory of molecular immunology and signal transduction-(GIGA-ULg). ORFs that were not available from the hORFeome V5.1 (BRAF, HRAS, ABL1, JAK2 and SMARCB1 and NOTCH1 genes), were purshassed from Genecopea and cloned by Gateway recombination technology (Invitrogen) using specific primers flanked with the following AttB1 and AttB2 Gateway sites: 5' - GGGGACAACTTTGTACAAAAAAGTTGGC ATG-3' (AttB1) and 5' - GGGGACAACTTTGTACAAG AAAGTTGA-3' (AttB2). These constructions were verified by PCR and sequencing.

Inserts from pDONR223 were transferred by LR cloning (Invitrogen) into different destination vectors: pADdestCYH and $\mathrm{pDB}$-dest the $\mathrm{Y} 2 \mathrm{H}$ expression vectors, and pDEST1899 (flag tag), pDEST491 (YFP-tag) and pDESTmcherry for mammalian expression studies.

\section{High-throughput yeast Two-hybrid (HT-Y2H)}

We used the hORFeome version 5.1, a collection of human ORFs cloned from the Mammalian Gene Collection (MGC) resource, representing a resource of ORFs that can be transferred easily to any Gateway compatible destination vectors. This collection contains 15483 ORFs representing almost half of the human genome. They are cloned into the pAD-dest-CYH and pDB-dest encoding the yeast Gal4 Activating and DNA-binding domains, respectively. The resulting individual clones were transferred into MATa Y8800 (pAD) and MAT $\alpha$ Y8930 (pDB) $S$. cerevisiae strains. Twenty-one selected ALL-genes were screened for interactions with the hORFeome V5.1 as described in [27].

One pool of $21 \mathrm{AD}$ of selected genes into Y8800 yeast strain was mated to each of the 15483 dB-ORFs Y8930 of the hORFeome v5.1 and each of ALL-genes-DB into Y8930 yeast strain was mated to 165 pools of 94 ADORFs of the hORFeome v5. One Y2H screening was performed in the reciprocal orientation, as described in [27]. Positive colonies for the GAL1:: HIS3 and GAL1:: ADE2 selective markers but negative for autoactivation were selected for PCR-amplification (Zymolyase $20 \mathrm{~T}$ from Seikagaku Biobusiness, and Platinum ${ }^{\bullet}$ Taq DNA Polymerase from Invitrogen) and identification of interacting proteins by sequencing of the respective AD- and DB-ORFs.

\section{Luciferase reporter assays}

Cells were seeded in 24-well plates and transfected with 300 ng of either TP1 luciferase reporter plasmid (TP1-luc) or CBF1 reporter plasmid (CBF1-luc) and $30 \mathrm{ng}$ of renilla Luciferase (R-Luc). Twenty-four hours post-transfection luciferase activity was measured in cell lysates.

U2OS N1-Gal4 cells they were transfected $300 \mathrm{ng}$ of Gal4-firefly luciferase and $30 \mathrm{ng}$ R-Luc reporter plasmid. After 24 h, K562 cells expressing Notch ligands DLL4 or K562 control cells were added to the transfected cells in the presence of tetracycline $(2 \mu \mathrm{g} / \mathrm{mL})$. After $24-\mathrm{h}$ of coculture, luciferase activity was measured in cell lysates.

Cell lysis and luciferase assays were performed in triplicate using Dual-luciferase reporter assay system from Promega. Luciferase measurements were performed in 96 well plates using DLR automated machine. Firefly luciferase values were normalized to R-luc values and calculated ratio represent luciferase activity.

\section{Immunofluorescence and confocal microscopy}

HeLa Notch1 1 E-eGFP cells were seeded onto coverslips in 24-well plates and transfected with $1 \mu \mathrm{g}$ of EXT1mcherry plasmid using lipofectamine2000 (Invotrogen). Twenty-four hours post-transfection, cells were washed in warm PBS, fixed in 3,7 \% PBS-paraformaldehyde for $20 \mathrm{~min}$ at room temperature, washed 3 times with PBS, 
and mounted on glass coverslips using ProLong Gold Antifade montant with DAPI (life technologies).

Slides were examined by confocal microscopy using the Nikon A1R confocal system and images processed with the IMAGI software.

\section{Protein complementation assay (PCA)}

NICD and FBXW7 were cloned in pN1Gluc vector and pN2Gluc vectors respectively (for Gaussia luciferase 1 and 2) using the Gateway cloning technology. HEK293 cells were seeded in 24 well-plates at a concentration $5.10^{4}$ cell/well, then transfected with GL1 or/and GL2 plasmids and $24 \mathrm{~h}$ post-transfection, luciferase activity was measured on lysates transferred into 96-well plate using and automated machine DLR with Renilla luciferase substrate. Normalized luciferase ratio was calculated as follows: NLR = luciferase value GL1 + GL2/(luciferase value GL1 + luciferase value GL2). An interaction is considered positive or validated when NLR $\geq 3.5$. Cell lysis and luciferase assays were performed in triplicate for each condition.

\section{EXT1 silencing in zebrafish}

Transgenic zebrafish line Tp1bglob:eGFP line [28] were maintained according to EU regulations on laboratory animals. Knockdown experiments were performed by injecting embryos at the one- to two-cell stage with 10 ng of single splice-blocking morpholino designed specifically for both EXT1 a and b orthologs.

\section{RNA sequencing}

Total RNA was extracted from HeLaN1 $\Delta$ E-eGFP cells (siCTRL, siEXT1, siFBXW7), quantified and tested for RNA quality controlled using Agilent 2100 bioanalyzer using the Eukaryote Total RNA Nano assay. Total RNA strands were used to generate libraries and sequenced by HiSeq2000 sequencer.

\section{Statistical analysis}

Graph values are presented as mean $+/$ - standard deviation, calculated on at least three independent experiments. Unless stated otherwise, significance was determined using a two-tailed Student's $t$-test (comparison of means). $P$-value thresholds are depicted as follows; " $: p<0.05 ;{ }^{* * *}: p<0.01$; ${ }^{* * * *}: p<0.001$ and ${ }^{* * * * *}: p<0.0001$.

To prioritize ALL-genes, we combined the ranking from separate results (rank per number of mutation, rank per number of samples, rank per degree) by using order statistics. First, ranks are divided by the total number of ranked genes and we calculated the $\mathrm{Q}$ statistic [29], which represents the probability of obtaining the observed ranks $r$ by chance, calculated using joint cumulative distribution of order as:

$$
\begin{aligned}
Q\left(r_{1}, r_{2}, \ldots, r_{N}\right) & =N ! V_{N} \\
V_{0} & =1, \\
V_{k} & =\sum_{i=1}^{k}(-1)^{i-1} \frac{V_{k}-i}{i !} r_{N-k+1}^{i}
\end{aligned}
$$

Where ri is the rank ratio for result $\mathrm{i}, \mathrm{N}$ is the number of genes used.

\section{Results and Discussion}

\section{Mutations associated to ALL in cancer gene census}

In order to identify cancer genes associated with acute lymphoblastic leukemia (ALL), we searched the COSMIC database version 71 and collected all available information about mutated genes in ALL samples. COSMIC V71 contains over 1,058,292 tumor samples containing over 2,710,449 coding mutations in 28,977 genes [1]. We found more than 2500 mutations in coding sequences of 366 genes that were reported in 36,909 ALL samples. In the COSMIC database, a set of 572 genes whose mutations are causally linked to oncogenesis, are called human Cancer Gene Census [30]. This set includes 140 genes well accepted as "cancer driver genes" because mutations in those genes directly promote tumorigenesis [2]. In ALL samples, we found that $20 \%$ of the cancer gene census is affected by mutations in coding regions of 116 genes (Additional file 1: Table S1A). This high number of mutated genes is not due to over representation of ALL samples in COSMIC, as ALL samples count for about $3 \%$ of tumor samples compiled in the COSMIC V71 (Fig. 1a). The "ALL-genes" set contains 74 wellknown driver genes including 35 oncogenes and 39 tumor suppressor genes (TSG) (Additional file 1: Table S1B).

For each ALL-gene we extracted the number of samples as well as the number of distinct mutations. Figure $1 \mathrm{~b}$ represents the top 20 frequently mutated genes among the 116 ALL-genes. Each gene that has at least 2 distinct mutations observed in at least 556 different samples, from a total of 36,909 ALL samples was examined. Seven genes were found mutated in more than $5 \%$ of ALL samples, including genes encoding for FLT3 (9.9\%), NRAS (6.14\%), JAK2 (6.08 \%), NOTCH1 (5.87 \%), IKZF1 (5.67 \%), KRAS (5.52 \%) and PTPN11 (5.32 \%) (Fig. 1b and c). We also ranked ALL-genes according to the number of distinct mutations found in ALL samples (Fig. 1b and d). The top ranked gene was $\mathrm{NOTCH} 1$ with 595 distinct mutations mostly found in its heterodimerization (HD) domain (63\% of mutations) and in its proline, glutamic acid, serine, threonine-rich (PEST) domain (27 \% of mutations) (Additional file 2: Figure S1). Mutations in the HD domain that enhance NOTCH1 cleavage and nuclear translocation of the intracellular NOTCH1 protein (ICN), and mutations in the PEST domain that result in the stabilization of ICN, are gain-of-function mutations affecting the transcriptional activation of Notch1-target genes. The majority 


\section{a}

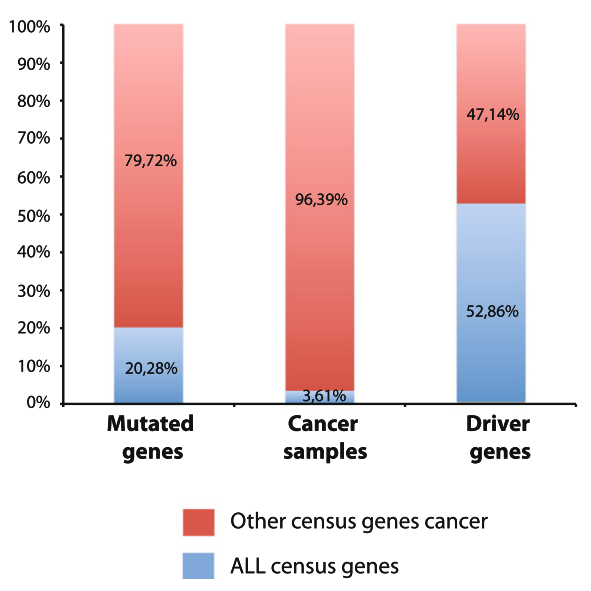

C

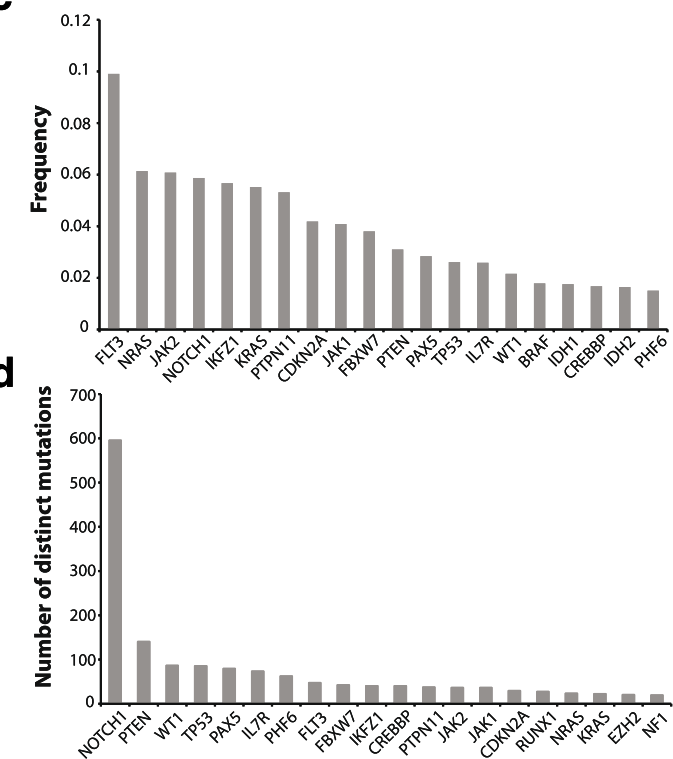

b

\begin{tabular}{|c|c|c|c|c|c|}
\hline $\begin{array}{c}\text { Gene } \\
\text { name }\end{array}$ & $\begin{array}{l}\text { Number of } \\
\text { samples }\end{array}$ & $\begin{array}{l}\text { Proportion } \\
\text { of samples }\end{array}$ & $\begin{array}{l}\text { Rank per } \\
\text { number of } \\
\text { samples with } \\
\text { mutations }\end{array}$ & $\begin{array}{l}\text { Distinct } \\
\text { mutations } \\
\text { per gene }\end{array}$ & $\begin{array}{l}\text { Rank per } \\
\text { distinct } \\
\text { mutations }\end{array}$ \\
\hline FLT3 & 3656 & $9,91 \%$ & 1 & 47 & 8 \\
NRAS & 2266 & $6,14 \%$ & 2 & 23 & 17 \\
JAK2 & 2244 & $6,08 \%$ & 3 & 36 & 13 \\
NOTCH1 & 2167 & $5,87 \%$ & 4 & 595 & 1 \\
IKFZ1 & 2093 & $5,67 \%$ & 5 & 40 & 10 \\
KRAS & 2037 & $5,52 \%$ & 6 & 22 & 18 \\
PTPN11 & 1963 & $5,32 \%$ & 7 & 37 & 12 \\
CDKN2A & 1545 & $4,19 \%$ & 8 & 29 & 15 \\
JAK1 & 1509 & $4,09 \%$ & 9 & 36 & 14 \\
FBXW7 & 1404 & $3,80 \%$ & 10 & 42 & 9 \\
PTEN & 1145 & $3,10 \%$ & 11 & 140 & 2 \\
PAX5 & 1049 & $2,84 \%$ & 12 & 79 & 5 \\
TP53 & 964 & $2,61 \%$ & 13 & 85 & 4 \\
IL7R & 955 & $2,59 \%$ & 14 & 73 & 6 \\
WT1 & 797 & $2,16 \%$ & 15 & 86 & 3 \\
BRAF & 660 & $1,79 \%$ & 16 & 7 & 34 \\
IDH1 & 649 & $1,76 \%$ & 17 & 5 & 43 \\
CREBBP & 619 & $1,68 \%$ & 18 & 40 & 11 \\
IDH2 & 607 & $1,64 \%$ & 19 & 2 & 73 \\
PHF6 & 556 & $1,51 \%$ & 20 & 62 & 7 \\
\hline
\end{tabular}

Total of samples $=36909$

e

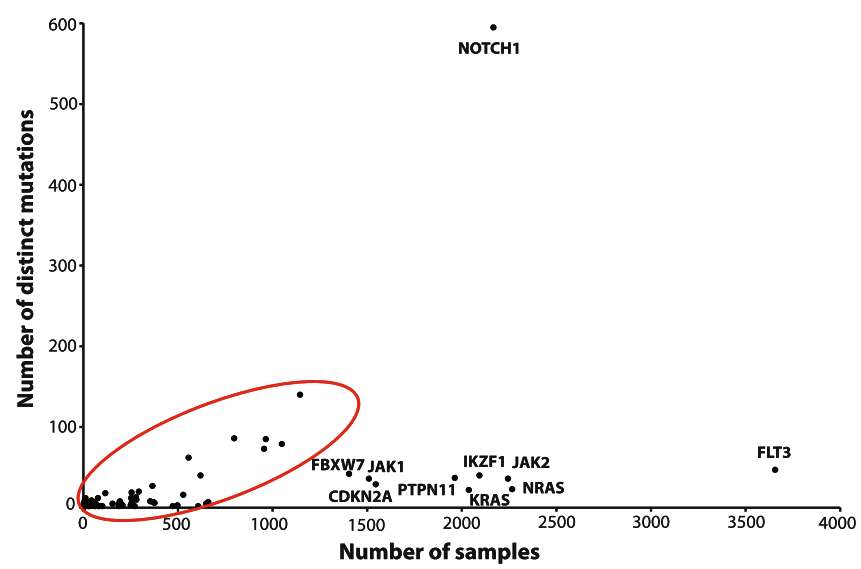

Fig. 1 a Distribution of ALL census genes and other census genes according to number of mutations and number of samples and their distribution among driver genes. Red and bleu bars represent ALL census genes and other cancer census genes respectively (b) Mutations associated to ALL in cancer gene census. Frequency of mutations in the top 20 genes (36,909 ALL samples). The number and proportion of ALL samples in which gene mutations were detected are represented. c The 20 most frequently mutated genes in ALL samples; X-axis represents the proportion of samples where mutations were reported. $\mathbf{d}$ Number of distinct mutations per genes in ALL samples; $X$-axis represents the number of distinct mutations found in the coding sequences and Y-axis the top 20 genes with higher number of distinct mutations. e Occurrence of mutations per gene in ALL samples. Data source: COSMIC database

of these activating mutations were found in human $\mathrm{T}$ lymphocytes ALL (T-ALL) samples, as previously reported [31]. Other highly mutated ALL-genes include PTEN (140 distinct mutations) WT1 (86 distinct mutations), TP53 (85 mutations), PAX5 (79 mutations), and IL7R (73 mutations) (Fig. 1d).

For the majority of ALL-genes, the number of distinct mutations per gene correlated with the number of mutated samples (Fig. 1e, red circled), suggesting that a number of somatic mutations occurred randomly during oncogenesis, as previously observed for other types of cancers such as ovarian carcinoma or acute myeloid leukemia [32]. Another set of eight genes (FBXW7, CDKN2A, PTPN11, $I K Z F 1, J A K 1, J A K 2, K R A S$ and NRAS) exhibit an average of 33 mutations in 1500-2500 examined ALL samples (Fig. 1e). These genes are characterized by similar mutations occurring in distinct ALL samples, suggesting their potential roles in clonal expansion of ALL. Two genes are outliers, and display many more mutations (NOTCH1) or are mutated in many more samples than average (FLT3). These larger numbers reflect the high rate of NOTCH1 mutations specifically in T-ALL 
samples (99,9 \% of NOTCH1 mutations); and the involvement of FLT3 in childhood ALL, as previously described [33]. Interestingly, NOTCH1 and FLT3 mutations, mostly localized in two functional domains (HD and PEST for NOTCH1, juxtamembrane (JM) and tyrosine kinase (TKD) for FLT3) (Additional file 2: Figure S1), are found respectively in 1897 and 723 different patients (Fig. 1b), without co-occurrence in examined ALL samples (Fig. 2b).

\section{Interconnections between ALL-gene products}

To analyze the connectivity between ALL-gene products, we collected protein-protein interactions (PPI) data from three databases: BioGRID [25], HPRD [24] and IntAct [23] and filtered all reported interactions between the 116 ALL-gene products. Figure 2a shows that 63 out 116 ALL-gene products are interconnected. We then prioritized ALL-gene products based on their degree of interconnectivity (Additional file 1: Table S2B). One of the top interconnected ALL-proteins is beta-catenin (encoded by CTNNB1 gene), which is a central hub in the $\mathrm{Wnt} / \beta$-catenin signalling pathway and plays a crucial role normal haematopoiesis [34]. It has been shown that $50-85 \%$ of the childhood T-ALL patients overexpress $\beta$-catenin [35], further supporting our finding that $\beta$ catenin is an important hub in ALL. Other Wnt/ $\beta$-catenin signaling pathway members such as APC, TCF3 and TCF7L2 interacting with $\beta$-catenin, are part of our ALL-gene product set and were previously found differentially expressed in T-ALL patients [35].
Another example is PIK3R1 with 8 partners including PIK3CA that interacts with additional 4 ALL-gene products. PI3K members are essential effectors in the PI3K/ $\mathrm{AKT} / \mathrm{mTOR}$ signaling pathway, which is activated in a number of ALL samples [36]. Another example is ABL1 that interacts with 7 partners. The BCR-ABL1 fusion is the driver chromosomal rearrangement in chronic myeloid leukemia (CML) [37] and is also found in more than $20 \%$ of ALL patients [38]. Mutations in ABL1 gene were associated to different types of cancer [39].

\section{Co-occurrence of mutations in ALL-genes}

We then explored the relationship between interacting genes based on the occurrence of mutations in the same ALL samples. We showed that, in addition to biophysical interactions, several ALL-gene products are mutated in the same patient samples, suggesting several ways of deregulating cancer pathways (Fig. 2b). As shown on Fig. 2b, our analysis revealed that ALL samples could be classified into 4 distinct groups of affected pathways, based on co-occurrence of mutations in important cancer driver genes: PI3K/AKT and NOTCH pathways, JAK and RAS pathways, Wnt/ $\beta$-catenin and the cell cycle, and the transcriptional regulation pathways. Interestingly, protein phosphatases PTEN and PTPN11, and proteins important in genome maintenance and chromatin modification P53 and CREB binding protein are centrals and connect with deregulated pathways through different set of mutations (Additional file 1: Table S3).

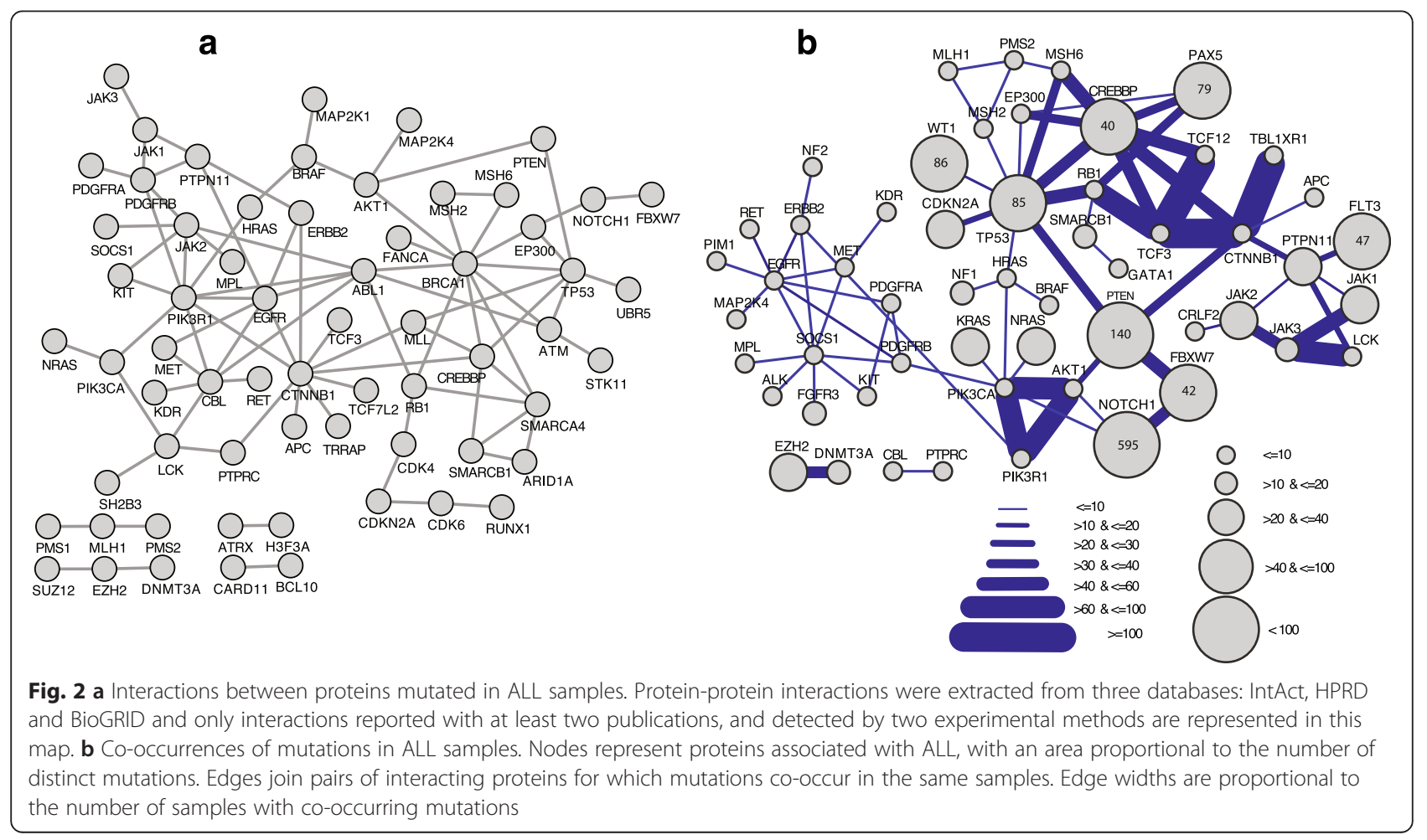


Combining all the above criteria: frequency of mutations in individual cancer genes in ALL samples, number of distinct mutations and their pattern in ALL-gene (Fig. 1 and Additional file 2: Figure S1), interconnections between ALL-gene products and co-occurrence of mutated genes in the same samples (Fig. 2), we prioritized ALL-genes and suggest that TP53, NOTCH1, CREBBP, PTEN, EGFR, JAK2, ABL1, PTPN11, CBL and $E P 300$ are the top 10 ALL driver genes (Additional file 1: Table S4).

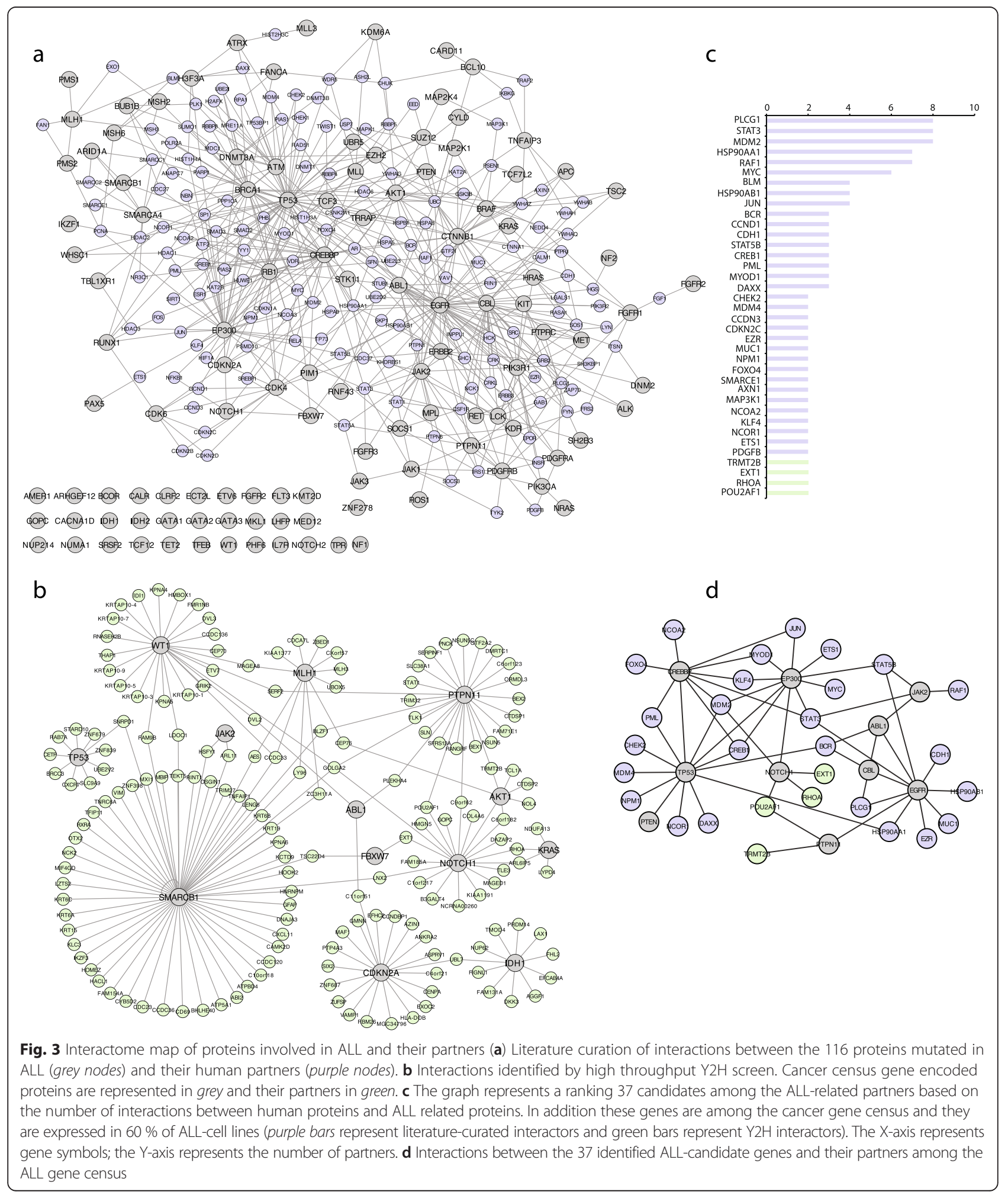


a

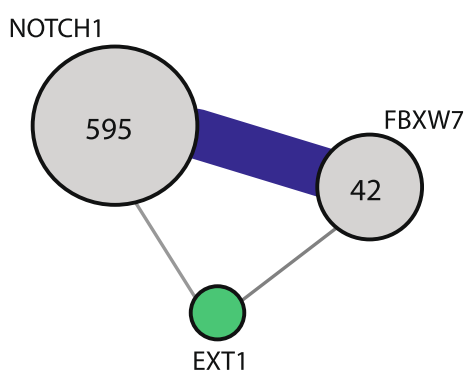

b
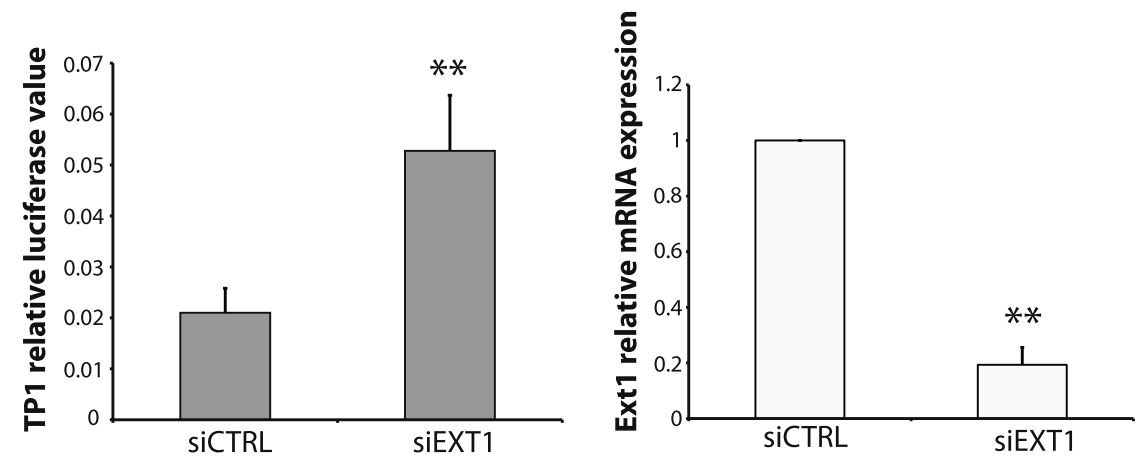

C
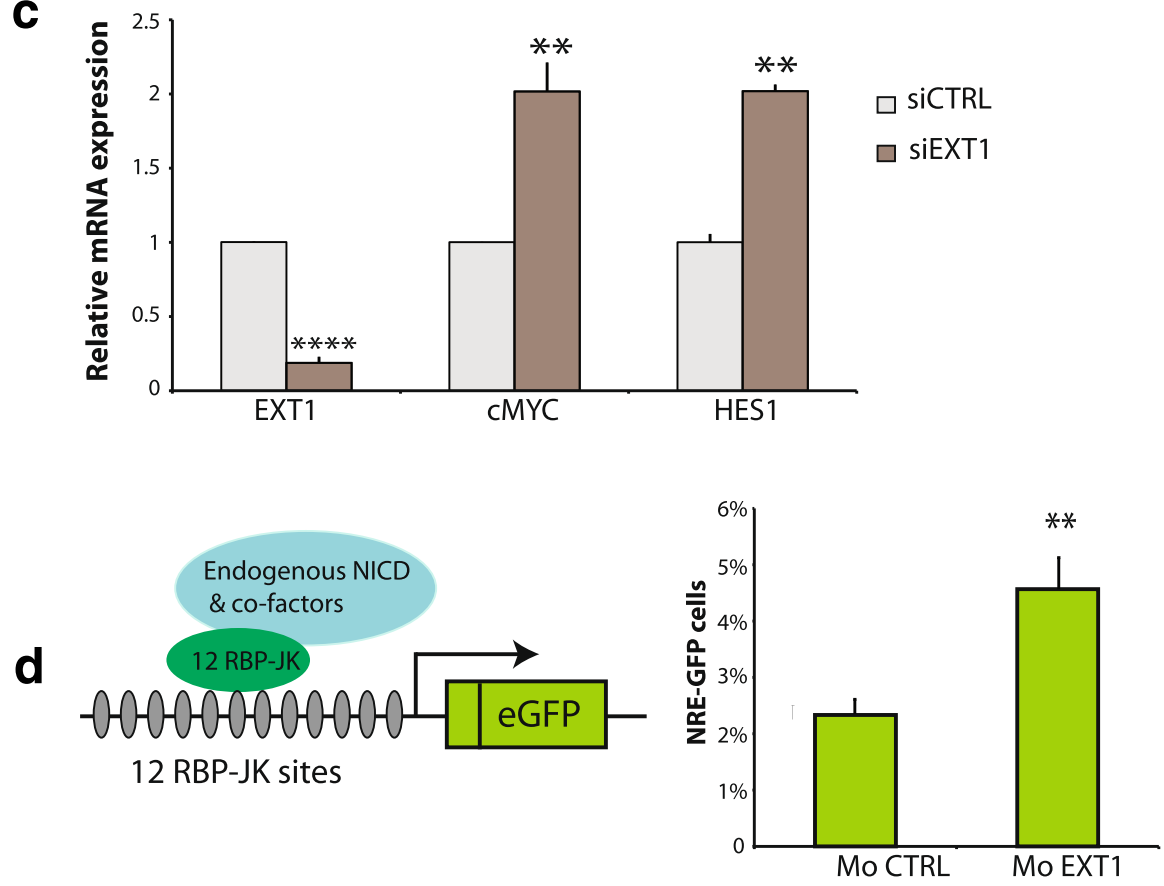

Fig. 4 EXT1 depletion promotes NOTCH1 transcriptional activity. a Interactions between NOTCH1, EXT1 and FBXW7. Grey nodes represent proteins associated with ALL, with an area proportional to the number of distinct mutations. Bleu edges join pairs of interacting proteins for which mutations co-occur in the same samples. Edge widths are proportional to the number of samples with co-occurring mutations. The green node represent EXT1 and grey edges interactions identified in $\mathrm{Y} 2 \mathrm{H}$ (b) Luciferase reporter assay using TP1-luciferase construct in HeLa Notch $\triangle \mathrm{E}$-eGFP cell lines transfected with EXT1 siRNA or control siRNA as indicated. The relative luciferase values are normalized using a Renilla luciferase construct. Knock-down of EXT1 was analysed by qPCR. $\mathbf{c}$ mRNA expression levels of Notch1 target genes; CMYC and EXT1 analysed by qPCR following EXT1 Knock down. $\mathbf{d}$ A zebrafish transgenic line Tg (Tp1bglob:eGFP) um13, reporter for Notch1 transcriptional activity, were treated with control or Ext1 a and c ortholog-targeted morpholinos. Left panel represents TP1 bglob:hngb1-eGFP construct. The graph represents the percentage eGFP cells sorted by FACS. Data represent the means \pm SEs of three independent experiments 
Functional associations between ALL-gene products and their partners in the human proteome

We hypothesized that the ALL-genes set is not limited to mutated genes in ALL samples, but could be extended to functional related genes and their products. In order to identify ALL-gene products interactors, we filtered from 3 different PPI databases (BioGRID, HPRD and IntAct), proteins that interact with at least 2 of the 116 ALL-gene products (Additional file 1: Table S5A). The obtained interactome map (Fig. 3a) shows that inter-connected ALL-gene products have also several common partners, prioritized according to the number of interacting ALLgene products (Additional file 1: Table S5B). PPI stored in databases are curated from the literature and some proteins such as P53, BRCA1 or ATM heavily studied with hundreds of publications, have more PPI reported than others that are not studied with equal intensity. Previous studies suggested that unbiased PPI mapping allow characterization of overlooked PPI and identification of unknown diseases-related candidates [10]. Our previous interactome analysis for the ALL-gene products derive from database interrogations, we then performed an experimental yeast-hybrid $(\mathrm{Y} 2 \mathrm{H})$ unbiased PPI detection assay using a set of ALL-genes and the human
ORFeome collection. We identified 193 interactions between 13 ALL gene products and 168 human partners. This experiment confirmed our observations using literature curated interactions, that interconnected ALL-gene products are also connected through several common partners in complex macromolecules (Fig. 3b). We identified several novel central hubs such as GOLGA2 that interacts with ALL-gene products NOTCH1, SMARCB1, PTPN11 and WT1, and CDC33, which is a common interactor of ALL-gene products MLH1, QT1, and SMARCB1 (Fig. 3b, Additional file 1: Table S4B).

As suggested by other studies, interconnected proteins are more associated with common diseases than expected by chance [40] and the same cancer driver genes are often involved in different cancer types, as evidenced by several examples [2]. To identify novel ALL-gene candidates through a "guilt-by-association" prediction, we prioritized ALL-gene products interactors using three criteria: (1) the number of ALL-gene products partners, (2) their implications in other types of cancer and (3) their expression in 24 common ALL cell lines. In total, we identified 37 ALL-gene products interactors that could be considered as ALL-associated candidates (Fig. 3c and d).

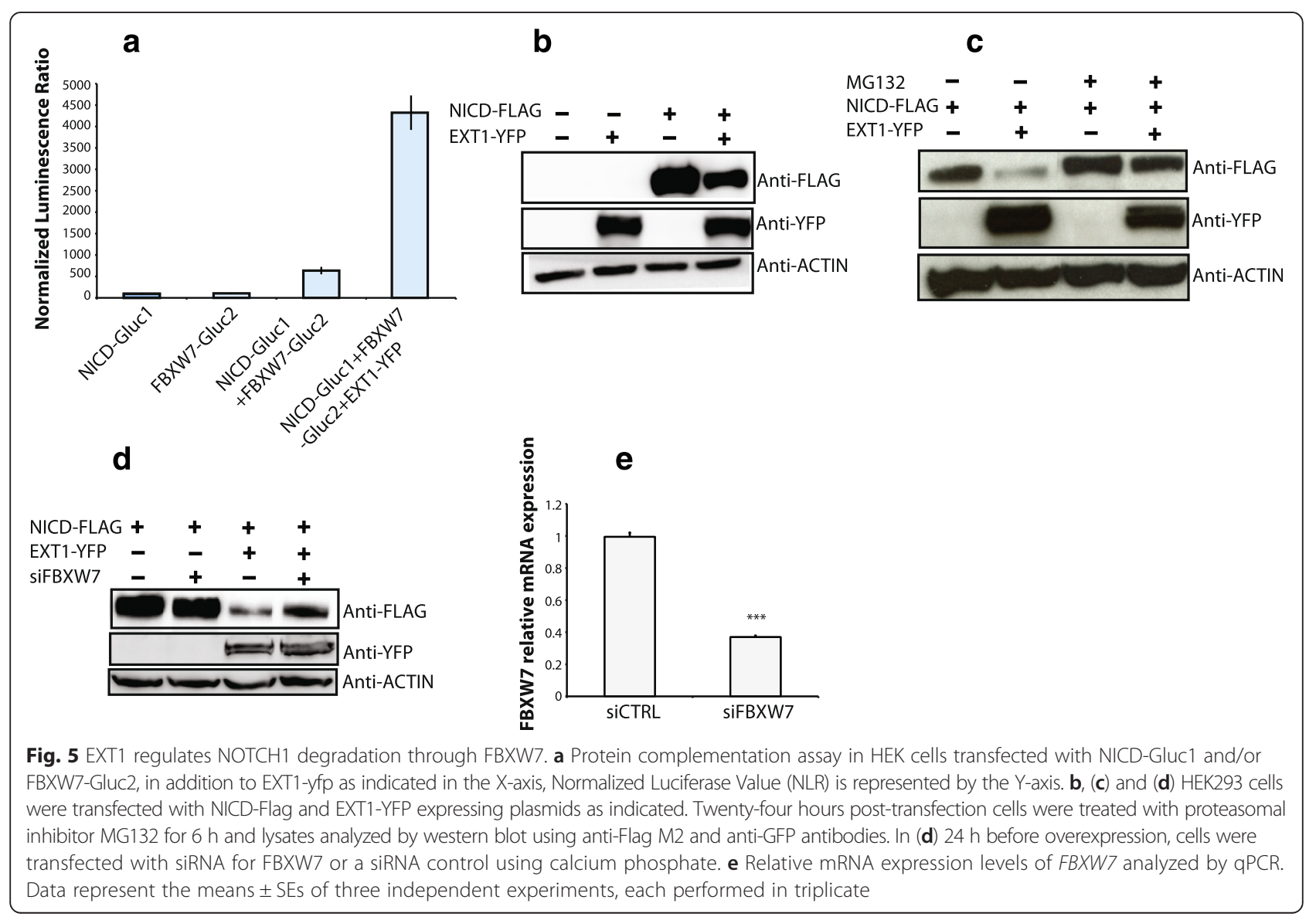


EXT1 is functionally associated with the notch pathway through its interaction with NOTCH1 and FBXW7

The following example illustrates the validity of combining interactome approaches and gene mutations characterization to identify specific cancer type-related genes. Exostosin glycosyltransferase 1 (EXT1) is an endoplasmic reticulum transmembrane protein frequently mutated in multiple osteochondromas [41-43]. We identified EXT1 as a common interactor of two ALL-gene products NOTCH1 and FBW7 (Figs. $3 \mathrm{~b}$ and $4 \mathrm{a}$ ). We then investigated the potential functional interplay between EXT1 and the NOTCH pathway. Using NOTCH1 transcriptional-responsive luciferase reporter assay, we showed that depletion of EXT1 using small interfering RNA increased NOTCH transactivation activity in different cell lines (Fig. 4b and Additional file 3: Figure S3a). We also showed that depletion of EXT1 increases mRNA expression levels of two important NOTCH1-target genes: HES1 and $M Y C$ (Fig. 4c). Consistent with this finding, over-expression of EXT1 inhibits NOTCH1-transactivation in different cell

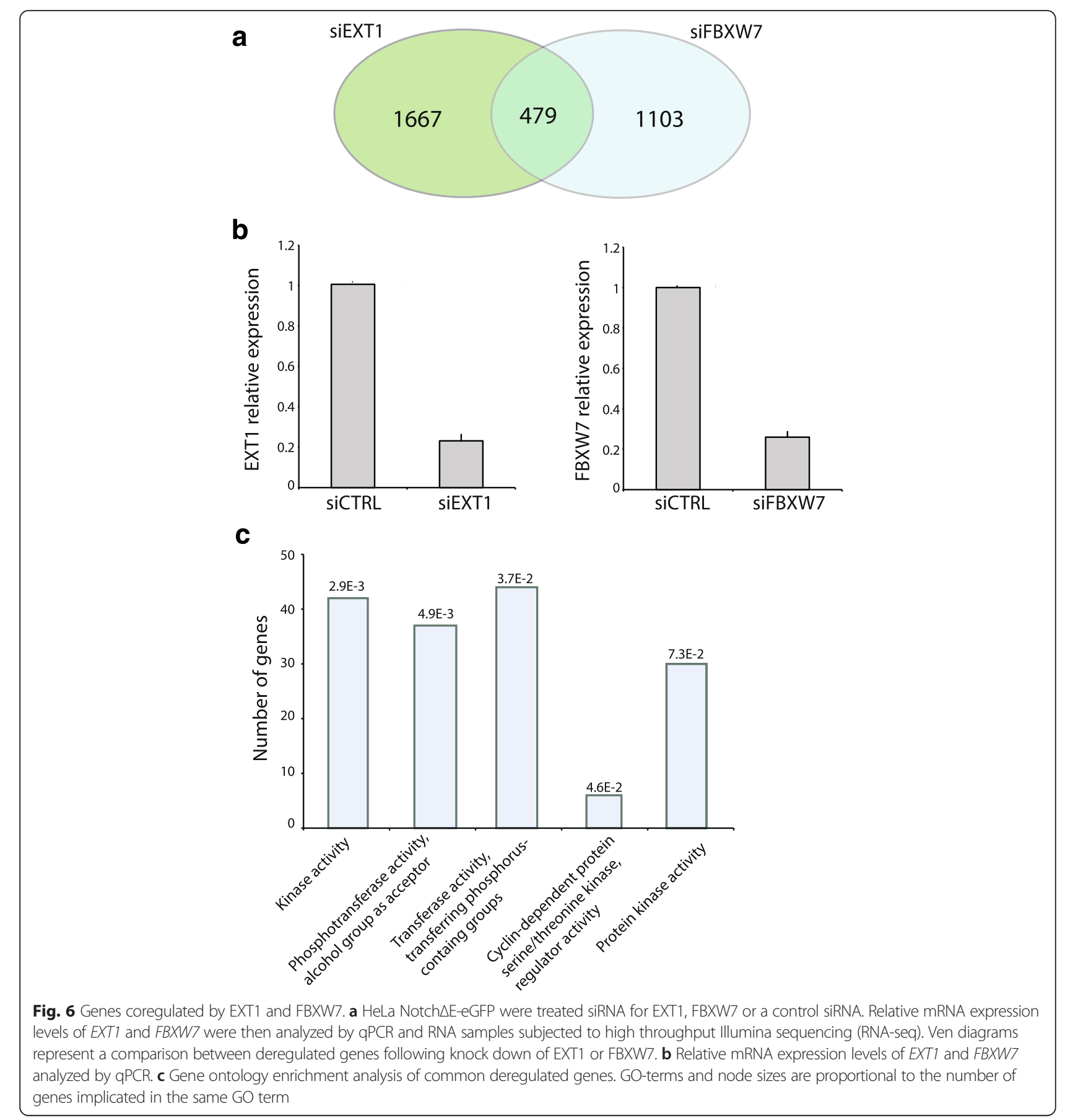


lines (Additional file 4: Figure S2) and correlates with a reduction of NICD protein levels (Additional file 5: Figure S4). We confirmed the effect of EXT1 on NOTCH1 pathway using a zebrafish in vivo model. We treated transgenic zebrafish line Tg (Tp1bglob:eGFP) um13 expressing fluorescent marker eGFP under the control of a Notchresponsive element TP1, with morpholinos targeting EXT1a and b zebrafish orhologs. As shown on Fig. 4d, we observed a $40 \%$ increase of NOTCH1 activity following depletion of EXT1 zebrafish orthologs (Fig. 4d).

FBXW7 is an E3 ubiquitin ligase regulating NOTCH1 proteasomal degradation [44]. To determine whether EXT1 interferes with FBXW7-NOTCH1 association, we first showed that the interaction between NOTCH1 and FBXW7 was dramatically enhanced in the presence of EXT1 (Fig. 5a). Then, we showed that, in the presence of EXT1, the level of NOTCH1 intracellular domain (NICD) is reduced in a proteasome-dependent manner (Fig. 5b and c: compare presence and absence of MG132 proteasome inhibitor). Interestingly, we also showed that reduced levels of NICD in the presence of EXT1 are FBXW7-dependent (Fig. 5d). To more deeply analyze the functional relationship between EXT1 and FBXW7 in regulating cellular homeostasis, a function well known for FBXW7 [44], we performed a genome-wide analysis of the transcriptome of HeLa-NICDdeltae-GFP [45] depleted for EXT1 or FBXW7. We identified 479 mRNAs co-regulated by both EXT1 and FBXW7, which represent more than $30 \%$ of FBXW7 targets (Fig. 6a). GO analysis of these EXT1/FBXW7 co-regulated genes finally indicated a significant enrichment in genes encoding for kinases including cyclin-dependent and MAP kinases (Fig. $6 \mathrm{c}$ and Additional file 1: Tables S6). Together, these results suggest that EXT1 is functionally linked to FBXW7, probably through priming kinases and substrates such as Notch1 towards proteasomal degradation.

\section{Conclusion}

The sequencing of different cancer genomes allows identification and characterization of mutated genes in cancer samples. However, the development of genome-based therapies requires greater knowledge of the specific driver genes implicated in diverse cancer types and subtypes. In this study, we have analyzed mutated genes found in ALL samples collected in the Sanger COSMIC database. We proposed a list of genes more likely involved in ALL by combining the frequency of mutations in ALL samples, the number and pattern of distinct mutations, and the interconnectivity between their products that determine specific affected signaling pathways. We finally propose that novel ALL-gene candidates can be identified based on their functional association with well-known cancer genes. Finally, we demonstrated that EXT1, a tumor suppressor not previously linked to ALL, is involved in the regulation of the $\mathrm{NOTCH}$ pathway trough its dual interaction with NOTCH1 and FBXW7.

\section{Additional files}

Additional file 1: Supplementary Tables. (XLSX 1285 kb)

Additional file 2: Figure S1. Distribution of mutations in $A L L$ patients for NOTCH1, FLT3 and FBXW7. Schematic representation for each protein and its domains, the number of ALL samples with mutations localizations and its percentage for each protein are represented above each domain. Notch1 domains: Epidermal growth factor repeats (EGF repeats), Lin12 and Notch repeats (LNR), heterodimerization domain (HD), transmembrane domain (TD), RBP-Jk-associated module (RAM), ankyrin repeats (ANK), transactivation domain (TAD), proline, glutamic acid, serine, threonine-rich domain (PEST). FLT3 domains: extracellular domain (EC), transmembrane domain (TM), juxtamembrane (JM), amino-terminal and carboxy-terminal kinase domains (TK1 and TK2 respectively), kinase domain (K1), (STK). FBXW7 domains: tryptophan-aspartic acid 40 repeat (WD). (EPS $781 \mathrm{~kb}$ )

Additional file 3: Figure S3. (a) EXT1 depletion promotes NOTCH1 transcriptional activity. (a) Luciferase reporter assay using TP1-luciferase construct in HEK293 cell lines transfected with EXT1 siRNA or control siRNA as indicated. The relative luciferase values are normalized using a Renilla luciferase construct. Knock-down of EXT1 was analysed by qPCR. (b) HEK293 cells were transfected with siRNA for EXT1 or a siRNA control using calcium phosphate. Twenty-four hours post-transfection cells were transfected with NICD-Flag expressing plasmid as indicated, and after $24 \mathrm{~h}$ lysates analyzed by western blot using anti-Flag M2 antibody. Knock-down of EXT1 was analyzed by qPCR. (c) HeLaNotch1 $\triangle$ E-eGFP cells were transfected with EXT1 siRNA or control siRNA as indicated. Forty two hours pottransfection, cells were treated with proteasomal inhibitor MG132 for $6 \mathrm{~h}$ and lysates were analyzed by western blot using a NICD antibody. Relative mRNA expression levels of EXT1 analyzed by qPCR. (d) HeLaNotch1 $\triangle E-e G F P$ cells were transfected with EXT1-YFP expressing plasmid as indicated. Eighteen hours post transfection, cells were treated with proteasomal inhibitor MG132 for $6 \mathrm{~h}$ and lysates were analyzed by western blot using a NICD antibody. (EPS 3297 kb)

Additional file 4: Figure S2. EXT1 inhibits Notch-1 transcriptional activation. (a) Luciferase reporter assay using TP1-luciferase construct in HEK293 and HeLa Notch $\triangle E-e G F P$ cell lines transfected with PSG5C control plasmid, NICD or EXT1 constructs as indicated. The relative luciferase values are normalized using a Renilla luciferase construct. (b) Luciferase reporter assay using Gal4-luciferase construct in U2OS N1-Gal4 cell line transfected with PSG5C control plasmid or EXT1 construct and COcultured with K562 control cells or K562 cells expressing Notch ligand DLL4 as indicated. Data represent the means \pm SES of three independent experiments, each performed in triplicate (EPS $752 \mathrm{~kb}$ )

Additional file 5: Figure S4. EXT1 reduces NOTCH1 levels in HelaNotch1 $\triangle$ EeGFP cells. Confocal images of HeLaNotch1 1 E-eGFP cells transfected with EXT1-mCherry during time-lapse experiment. Green and red labeling corresponds to NOTCH1-GFP and EXT1-mCherry proteins localizations, respectively. Arrows indicate cells in which overexpression of EXT1 induced a decrease in GFP fluorescence. (PDF 6860 kb)

\section{Acknowledgements}

We thank the GIGA-Research technology platforms (Interactome, Genomics, imaging, Flow cytometry, viral vectors and Zebrafish facility) for technical assistance. We also thank the COSMIC database for data curation.

\section{Funding}

This work was supported by the Fonds National de la Recherche Scientifique (FNRS) grants \# 7.4538.09/11/13 to JCT, the Fondation Belge contre le Cancer (FBC) grant \# HE2010 to FD, and the Fonds Leon Fredericq to SD.

\section{Availability of data and materials}

All datasets supporting the conclusions of this article are included within the main manuscript or in supplemental files. 


\section{Authors' contributions}

SD carried out interactomes screening and cell culture assays and drafted the manuscript. LH collected mutations and interactomes data and performed network analysis. DK and AD carried out some cell culture experiments. NS, RK, FD and JCT directed the study. JCT wrote the manuscript. All authors read and approved the final manuscript.

\section{Competing interests}

The authors declare that they have no competing interests.

\section{Consent for publication}

Not applicable.

\section{Ethics approval and consent to participate}

Not applicable.

\section{Author details}

'Laboratory of Protein Signaling and Interactions, Molecular Biology in Diseases Unit, GIGA-Research, University of Liège, Liège B-4000, Belgium. ${ }^{2}$ Laboratoire de Bioinformatique des Génomes et des Réseaux (BiGRe), Université Libre de Bruxelles (ULB), Bruxelles B-1050, Belgium.

\section{Received: 20 January 2016 Accepted: 19 May 2016}

Published online: 26 May 2016

\section{References}

1. Forbes SA et al. COSMIC: exploring the world's knowledge of somatic mutations in human cancer. Nucleic Acids Res. 2015:43:D805-11.

2. Vogelstein B et al. Cancer Genome Landscapes. Science. 2013;339:1546-58.

3. Hindorff $L A$ et al. Potential etiologic and functional implications of genomewide association loci for human diseases and traits. Proc Natl Acad Sci. 2009;106:9362-7

4. March $\mathrm{HN}$ et al. Insertional mutagenesis identifies multiple networks of cooperating genes driving intestinal tumorigenesis. Nat Genet. 2011;43: 1202-9.

5. Starr TK et al. A Transposon-Based Genetic Screen in Mice Identifies Genes Altered in Colorectal Cancer. Science (New York, NY). 2009:323:1747-50.

6. Takeda $\mathrm{H}$ et al. Transposon mutagenesis identifies genes and evolutionary forces driving gastrointestinal tract tumor progression. Nat Genet. 2015;47: $142-50$.

7. de Chassey B et al. Hepatitis C virus infection protein network. Mol Syst Biol. 2008:4:230.

8. Simonis $\mathrm{N}$ et al. Host-pathogen interactome mapping for HTLV-1 and -2 retroviruses. Retrovirology. 2012:9:26.

9. Rozenblatt-Rosen $\mathrm{O}$ et al. Interpreting cancer genomes using systematic host perturbations by tumour virus proteins. Nature. 2012:487:491-5.

10. Rolland T et al. A Proteome-Scale Map of the Human Interactome Network. Cell. 2014:159:1212-26.

11. Parsons DW et al. The genetic landscape of the childhood cancer medulloblastoma. Science (New York, NY). 2011:331:435-9.

12. Tomasetti C, Marchionni L, Nowak MA, Parmigiani G, Vogelstein B. Only three driver gene mutations are required for the development of lung and colorectal cancers. Proc Natl Acad Sci. 2015;112:118-23.

13. Shtivelman E, Lifshitz B, Gale RP, Canaani E. Fused transcript of abl and bcr genes in chronic myelogenous leukaemia. Nature. 1985;315(6020):550-4.

14. Maher CA et al. Transcriptome Sequencing to Detect Gene Fusions in Cancer. Nature. 2009:458:97-101.

15. Gough SM, Slape Cl, Aplan PD. NUP98 gene fusions and hematopoietic malignancies: common themes and new biologic insights. Blood. 2011; 118:6247-57

16. Torrano V, Procter J, Cardus P, Greaves M, Ford AM. ETV6-RUNX1 promotes survival of early $B$ lineage progenitor cells via a dysregulated erythropoietin receptor. Blood. 2011;118:4910-8.

17. Zhou M-h et al. Detection of ETV6 gene rearrangements in adult acute lymphoblastic leukemia. Ann Hematol. 2012;91:1235-43.

18. Tijchon E, Havinga J, Van Leeuwen FN, Scheijen B. B-lineage transcription factors and cooperating gene lesions required for leukemia development. Leukemia. 2013:27:541-52.

19. Seto M. Genomic profiles in B cell lymphoma. Int J Hematol. 2010;92:238-45.
20. Hajingabo $L J$ et al. Predicting interactome network perturbations in human cancer: application to gene fusions in acute lymphoblastic leukemia. Mol Biol Cell. 2014;25:3973-85.

21. Jeong H, Mason SP, Barabasi AL, Oltvai ZN. Lethality and centrality in protein networks. Nature. 2001;411:41-2.

22. Goh K-l et al. The human disease network. Proc Natl Acad Sci. 2007;104: 8685-90.

23. Kerrien $\mathrm{S}$ et al. IntAct-open source resource for molecular interaction data. Nucleic Acids Res. 2007:35:D561-5.

24. Mishra GR et al. Human protein reference database-2006 update. Nucleic Acids Res. 2006:34:D411-4

25. Breitkreutz B-J et al. The BioGRID Interaction Database: 2008 update. Nucleic Acids Res. 2008:36:D637-40.

26. Cline MS et al. Integration of biological networks and gene expression data using Cytoscape. Nat Protocols. 2007;2:2366-82.

27. Rual J-F et al. Towards a proteome-scale map of the human protein-protein interaction network. Nature. 2005:437:1173-8.

28. Parsons MJ et al. Notch-responsive cells initiate the secondary transition in larval zebrafish pancreas. Mech Dev. 2009;126:898-912.

29. Aerts $\mathbf{S}$ et al. Gene prioritization through genomic data fusion. Nat Biotech. 2006;24:537-44

30. Futreal PA et al. A census of human cancer genes. Nat Rev Cancer. 2004:4: $177-83$

31. Weng AP et al. Activating Mutations of NOTCH1 in Human T Cell Acute Lymphoblastic Leukemia. Science. 2004;306:269-71.

32. Kandoth $\mathrm{C}$ et al. Mutational landscape and significance across 12 major cancer types. Nature. 2013;502:333-9.

33. Kabir N, Rönnstrand L, Kazi J. FLT3 mutations in patients with childhood acute lymphoblastic leukemia (ALL). Med Oncol. 2013;30:1-3.

34. Serinsöz $E$ et al. Aberrant expression of $\beta$-catenin discriminates acute myeloid leukaemia from acute lymphoblastic leukaemia. Br J Haematol. 2004;126:313-9.

35. $\mathrm{Ng} \mathrm{OH}$ et al. Deregulated WNT signaling in childhood T-cell acute lymphoblastic leukemia. Blood Cancer Journal. 2014;4:e192

36. Zuurbier $L$ et al. The significance of PTEN and AKT aberrations in pediatric T-cell acute lymphoblastic leukemia. Haematologica. 2012;97:1405-13.

37. Ren $R$. Mechanisms of $B C R-A B L$ in the pathogenesis of chronic myelogenous leukaemia. Nat Rev Cancer. 2005;5:172-83.

38. Secker-Walker LM et al. Variable Philadelphia breakpoints and potential lineage restriction of bcr rearrangement in acute lymphoblastic leukemia. Blood. 1988;72:784-91.

39. de Oliveira GAP, Rangel LP, Costa DC, Silva JL. Misfolding, Aggregation, and Disordered Segments in c-Abl and p53 in Human Cancer. Frontiers in Oncology. 2015:5:97.

40. Vidal M, Cusick ME, Barabási A-L. Interactome Networks and Human Disease. Cell. 2011;144:986-98.

41. Simmons AD et al. A Direct Interaction Between EXT Proteins and Glycosyltransferases is Defective in Hereditary Multiple Exostoses. Hum Mol Genet. 1999;8:2155-64.

42. Philippe $C$ et al. Mutation screening of the EXT1 and EXT2 genes in patients with hereditary multiple exostoses. Am J Hum Genet. 1997;61:520-8.

43. Zhang F et al. Exome Sequencing and Functional Analysis Identifies a Novel Mutation in EXT1 Gene That Causes Multiple Osteochondromas. PLoS One. 2013;8:e72316.

44. Crusio KM, King B, Reavie LB, Aifantis I. The ubiquitous nature of cancer: the role of the SCF (Fbw7) complex in development and transformation. Oncogene. 2010:29:4865-73.

45. Huenniger $\mathrm{K}$ et al. Notch 1 signaling is mediated by importins alpha 3, 4, and 7 . Cell Mol Life Sci. 2010;67:3187-96. 